\title{
Prolonged PR Interval and Risk of Recurrence of Atrial Fibrillation After Catheter Ablation
}

\author{
Jin-Tao Wu, ${ }^{1} \mathrm{MD}$, Jian-Zeng Dong, ${ }^{2} \mathrm{MD}$, Cai-Hua $\mathrm{SANG},{ }^{2} \mathrm{MD}$, \\ Ri-Bo TANG, ${ }^{2} \mathrm{MD}$, and Chang-Sheng $\mathrm{MA},{ }^{2} \mathrm{MD}$
}

\begin{abstract}
SUMMARY
It has been demonstrated that a prolonged PR interval is associated with an increased risk of AF. However, the impact of a prolonged PR interval on recurrence of paroxysmal atrial fibrillation (AF) after catheter ablation is not clear. A total of 112 patients with a prolonged PR interval (PR > $200 \mathrm{~ms}$ ) (PPR group) and 112 age- and gender-matched control patients (on a 1:1 basis) with a normal PR interval (NPR group) were included in this study. AF recurrence was defined as the occurrence of confirmed atrial tachyarrhythmia lasting more than 30 seconds beyond 3 months after catheter ablation in the absence of any antiarrhythmic treatment. During a mean follow-up period of $10.9 \pm 5.5$ months (range, 3-18 months), 61 patients $(27.2 \%)$ developed recurrence of AF. The recurrence rate was higher in the PPR group than in the NPR group (33.9\% versus $20.5 \%$, respectively; $P=0.018)$. Cox regression analysis with adjustment for age, body mass index, valvular heart disease, left atrial diameter, and pulmonary vein isolation identified only a prolonged PR interval as an independent predictor of recurrence of AF (hazard ratio, 1.81; 95\% confidence interval, 1.07-3.05; $P=0.027$ ). Patients with a prolonged PR interval were at an increased risk of AF recurrence after catheter ablation. (Int Heart J 2014; 55: 126-130)
\end{abstract}

Key words: Atrial tachyarrhythmia

A trial fibrillation $(\mathrm{AF})$ is a common cardiac arrhythmia in the general population ${ }^{1)}$ and is associated with increased cardiovascular morbidity and mortality. ${ }^{2,3)} \mathrm{A}$ recent post hoc analysis of a Danish multicenter randomized trial (DANPACE trial) showed that a prolonged PR interval is associated with an increased risk of AF in patients with sick sinus syndrome. ${ }^{4)}$ These results are in accordance with the findings of a large community-based cohort study in which a PR interval longer than $200 \mathrm{~ms}$ was associated with a doubled risk of later AF compared with a shorter PR interval. ${ }^{5)}$ Catheter ablation has been established as an effective therapy for drug-refractory paroxysmal AF. However, there is still a risk of recurrence after ablation. Previous studies have examined some predictors of AF recurrence. ${ }^{6,7)}$ However, there is little information available about the impact of a prolonged PR interval on recurrence of $\mathrm{AF}$ after catheter ablation. In patients with a prolonged PR interval, most of the pathogenic factors for AF persist after ablation. Therefore, it is reasonable to hypothesize that a prolonged PR interval increases the risk of recurrence of AF. This retrospective study evaluated the impact of a prolonged PR interval on recurrence of AF after catheter ablation.

\begin{abstract}
Methods
Study subjects: We reviewed the records of 1593 consecutive patients with refractory symptomatic paroxysmal AF who had been prospectively entered into our database. All of these patients were referred to Beijing An Zhen Hospital, Capital Medical University for first-time circumferential pulmonary vein (PV) radiofrequency ablation from January 2008 to December 2012. The PR interval was considered to be prolonged if it was $>200 \mathrm{~ms}$ and to be normal if it was $\leq 200 \mathrm{~ms}$ as determined from a resting 12-lead electrocardiogram after all antiarrhythmic drugs had been discontinued for at least 5 half-lives and in the absence of $\beta$-blocker and calcium channel blocker use. Of the 1593 patients, 112 had a prolonged PR interval or first-degree atrioventricular block. These patients were enrolled in the study (PPR group) together with 112 age- and gender-matched patients (on a 1:1 basis) with a normal PR interval (PR $\leq 200$ ms) (NPR group). All patients provided written informed consent, and the study was approved by the institutional review board. Transesophageal and transthoracic echocardiography images were obtained prior to ablation to measure the left atrial (LA) anteroposterior diameter, left ventricular (LV) end-diastolic diameter, LV end-systolic diameter, and LV ejection fraction and to exclude the presence of intra-atrial thrombus.

Electrophysiological study and catheter ablation: The ablation
\end{abstract}

From the ${ }^{1}$ Department of Cardiology, Henan Provincial People's Hospital, Zhengzhou University, Zhengzhou and ${ }^{2}$ Department of Cardiology, Center for Atrial Fibrillation, Beijing Anzhen Hospital, Capital Medical University, Beijing, China.

Address for correspondence: Jian-Zeng Dong, MD, Department of Cardiology, Center for Atrial Fibrillation, Beijing Anzhen Hospital, Capital Medical University, Anzhen Road, Chaoyang District, Beijing 100029, China. E-mail: jz_dong@126.com

Received for publication August 11, 2013. Revised and accepted September 28, 2013

Released advance online J-STAGE March 14, 2014.

All rights are reserved to the International Heart Journal Association. 
Table I. Characteristics of Patients in the PPR and NPR Groups

\begin{tabular}{lccc}
\hline & PPR $(n=112)$ & NPR $(n=112)$ & $P$ \\
\hline Age $($ years) & $61 \pm 11$ & $61 \pm 11$ & 1.00 \\
Male, $n(\%)$ & $76(67.9 \%)$ & $76(67.9 \%)$ & 1.00 \\
BMI $\left(\mathrm{kg} / \mathrm{m}^{2}\right)$ & $25.9 \pm 3.1$ & $25.7 \pm 3.0$ & 0.623 \\
AF duration (years) & $6.3 \pm 4.3$ & $6.5 \pm 4.1$ & 0.780 \\
Heart rate (beart/minute) & $69 \pm 10$ & $68 \pm 9$ & 0.434 \\
DM, $n(\%)$ & $21(18.8 \%)$ & $10(8.9 \%)$ & 0.033 \\
Hypertension, $n(\%)$ & $55(49.1 \%)$ & $62(55.4 \%)$ & 0.349 \\
Heart failure, $n(\%)$ & $2(1.8 \%)$ & $3(2.7 \%)$ & 0.651 \\
Valvular heart disease, $n(\%)$ & $32(28.6 \%)$ & $15(13.4 \%)$ & 0.005 \\
CAD, $n(\%)$ & $2(10.7 \%)$ & $9(8.0 \%)$ & 0.492 \\
Left atrial diameter (mm) & $37.9 \pm 4.9$ & 0.025 \\
LVEDD (mm) & $39.3 \pm 5.9$ & $47.9 \pm 5.2$ & 0.502 \\
LVESD (mm) & $48.3 \pm 5.6$ & $31.7 \pm 4.6$ & 0.574 \\
Ejection fraction $(\%)$ & $32.0 \pm 4.5$ & $63.2 \pm 9.0$ & 0.731 \\
ACEI/ARB, $n(\%)$ & $62.9 \pm 9.4$ & $33(29.5 \%)$ & 0.318 \\
Statins, $n(\%)$ & $40(35.7 \%)$ & $22(19.6 \%)$ & 0.118 \\
Pulmonary vein isolation, $n(\%)$ & $32(28.6 \%)$ & $110(98.2 \%)$ & 0.651
\end{tabular}

BMI indicates body mass index; AF, atrial fibrillation; DM, diabetes mellitus; CAD, coronary artery disease; LVEDD, left ventricular end-diastolic diameter; LVESD, left ventricular end-systolic diameter; ACEI, angiotensin-converting enzyme inhibitors; and ARB, angiotensin receptor blockers.

procedure was performed in the postabsorptive state under conscious sedation. We employed the technique of circumferential PV ablation guided by 3-dimensional left atrial mapping, which was described previously in detail. ${ }^{8,9)}$ Briefly, the LA was explored using a trans-septal approach. The LA geometry was reconstructed with a $3.5-\mathrm{mm}$ tip ablation catheter (NaviStar ThermoCool; Biosense Webster, USA) in a CARTO system. Continuous irrigated radiofrequency ablation was performed along each PV antrum to encircle the ipsilateral PVs (target temperature, $43^{\circ} \mathrm{C}$; maximum power, $35 \mathrm{~W}$; infusion rate, $17 \mathrm{~mL} / \mathrm{min}$ ). The procedural endpoints were completeness of continuous circular lesions and electrical isolation of all PVs as identified by a decapolar circumferential mapping catheter (Lasso; Biosense Webster). If a typical atrial flutter had been documented before the procedure, the tricuspid isthmus responsible for this tachycardia was identified and ablated. Postablation management and follow-up: After the procedure, all patients received antiarrhythmic drugs if there were neither contraindications nor intolerance. If no recurrent atrial tachyarrhythmia occurred after 2 or 3 months, the drug treatment was discontinued. All asymptomatic patients were followed up with a 12-lead electrocardiogram and 24-hour Holter recordings at the 1st, 3rd, 6th, and 12th month and every 6 months beyond 1 year after the ablation procedure. If the patient was symptomatic, a new electrocardiogram or 24-hour Holter recording was obtained. Recurrence was defined as the occurrence of confirmed atrial tachyarrhythmia lasting more than 30 seconds (documented by electrocardiogram or Holter recordings) beyond 3 months after the catheter ablation in the absence of any antiarrhythmic treatment.

Statistical analysis: All analyses were performed with SPSS software version 17.0. Continuous data are presented as the mean \pm standard deviation. Univariate analysis to assess the predictive value of clinical variables on AF recurrence was performed using the unpaired independent-samples $t$-test for continuous variables and the $\chi^{2}$ test and Fisher's exact test if necessary for categorical variables. A Kaplan-Meier estimation with a log-rank test was performed for unadjusted analysis

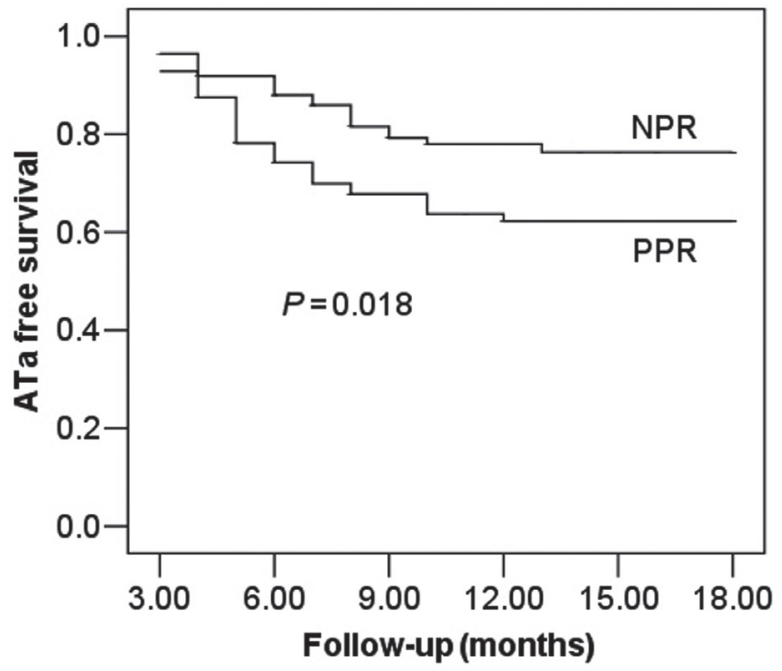

Figure. Kaplan-Meier curves showing recurrence in patients with prolonged and normal PR intervals. Patients with a prolonged PR interval had a higher incidence of recurrence of atrial tachyarrhythmia (ATa) than did patients with a normal PR interval $(33.9 \%$ versus $20.5 \%$, respectively; $P=$ 0.018 by log-rank test).

of the impact of a prolonged PR interval on recurrence of AF. Cox proportional hazards regression was employed to examine the risks for recurrence. Age, body mass index (BMI), valvular heart disease, LA diameter, and PV isolation were adjusted in the Cox analysis. All probability values were 2 -sided, and a $P$ of $<0.05$ was considered significant.

\section{Results}

Clinical characteristics of patients with prolonged and normal PR intervals: The clinical characteristics of patients in the PPR and NPR groups are shown in Table I. The prevalences of 
Table II. Characteristics of Patients With and Without Recurrence of AF

\begin{tabular}{lccc}
\hline & Recurrence $(n=61)$ & Non-recurrence $(n=163)$ & $P$ \\
\hline Age $($ years) & $62 \pm 11$ & $61 \pm 11$ & 0.347 \\
Male, $n(\%)$ & $43(70.5 \%)$ & $109(66.9 \%)$ & 0.605 \\
BMI $\left(\mathrm{kg} / \mathrm{m}^{2}\right)$ & $26.2 \pm 3.1$ & $25.7 \pm 3.0$ & 0.357 \\
AF duration (years) & $6.3 \pm 4.4$ & $6.4 \pm 4.1$ & 0.821 \\
DM, $n(\%)$ & $10(16.4 \%)$ & $21(12.9 \%)$ & 0.231 \\
Hypertension, $n(\%)$ & $35(57.4 \%)$ & $82(50.3 \%)$ & 0.346 \\
Heart failure, $n(\%)$ & $2(3.3 \%)$ & $3(1.8 \%)$ & 0.615 \\
Valvular heart disease, $n(\%)$ & $15(24.6 \%)$ & $32(19.6 \%)$ & 0.488 \\
CAD, $n(\%)$ & $7(11.5 \%)$ & $14(8.6 \%)$ & 0.509 \\
Left atrial diameter $(\mathrm{mm})$ & $39.0 \pm 5.6$ & $38.2 \pm 5.2$ & 0.281 \\
LVEDD $(\mathrm{mm})$ & $48.5 \pm 5.1$ & $48.0 \pm 5.4$ & 0.468 \\
LVESD (mm) & $31.6 \pm 4.8$ & $31.8 \pm 4.5$ & 0.714 \\
Ejection fraction $(\%)$ & $62.4 \pm 11.0$ & $63.3 \pm 8.7$ & 0.525 \\
ACEI/ARB, $n(\%)$ & $23(37.7 \%)$ & $50(30.7 \%)$ & 0.318 \\
Statins, $n(\%)$ & $13(21.3 \%)$ & $41(25.2 \%)$ & 0.550 \\
PPR, $n(\%)$ & $38(62.3 \%)$ & $74(45.4 \%)$ & 0.024 \\
Pulmonary vein isolation, $n(\%)$ & $59(96.7 \%)$ & $160(98.2 \%)$ & 0.503 \\
\hline
\end{tabular}

PPR indicates prolonged PR interval. For other abbreviations, see Table I.

Table III. Prolonged PR Interval and Recurrence of AF in Multivariate Analysis

\begin{tabular}{lcc}
\hline Variables & $P$ & HR $(95 \% \mathrm{CI})$ \\
\hline Age & 0.262 & $1.01(0.99-1.04)$ \\
BMI & 0.124 & $1.07(0.98-1.15)$ \\
Valvular heart disease & 0.453 & $1.25(0.69-2.27)$ \\
Left atrial diameter & 0.596 & $1.01(0.97-1.06)$ \\
Pulmonary vein isolation & 0.718 & $1.30(0.31-5.44)$ \\
PPR & 0.027 & $1.81(1.07-3.05)$ \\
\hline
\end{tabular}

HR indicates hazard ratio and CI, confidence interval.

diabetes mellitus and valvular heart disease were significantly higher in the PPR group than in the NPR group. The LA diameter was significantly larger in the PPR group than in the NPR group. Age, gender, AF duration, heart rate, LV ejection fraction, LV end-diastolic dimension, and LV end-systolic dimension were not significantly different between the 2 groups. The prevalences of hypertension, coronary artery disease, and medication use ( $\beta$-blockers, angiotensin-converting enzyme inhibitors/angiotensin receptor blockers, and statins) were not significantly different between the 2 groups.

Recurrence of $\mathrm{AF}$ in patients with a prolonged $\mathrm{PR}$ interval: During the mean follow-up period of $10.9 \pm 5.5$ months (range, 3-18 months), 61 patients $(27.2 \%)$ developed recurrence of AF. The recurrence rate was $33.9 \%$ in the PPR group and $20.5 \%$ in the NPR group $(P=0.018)$ (Figure). The characteristics of patients with and without recurrence are shown in Table II. Univariate analyses found that a prolonged PR interval was the only predictor of recurrence of AF. Cox regression analysis with adjustment for age, BMI, valvular heart disease, LA diameter, and PV isolation identified only a prolonged PR interval as an independent predictor of recurrence of AF (hazard ratio, $1.81 ; 95 \%$ confidence interval, $1.07-3.05 ; P=0.027$ ) (Table III).

\section{Discussion}

The main finding of the present study is that a prolonged
$\mathrm{PR}$ interval is an independent predictor of recurrence of AF in patients who have undergone catheter ablation for paroxysmal AF.

Several studies have demonstrated that a prolonged PR interval is associated with an increased risk of AF. ${ }^{4,5,10)}$ The exact mechanism for this is unknown. The PR interval is determined by the conduction time from the sinus node to the ventricles and thus integrates information about a number of sites in the conduction system of the heart. Prolongation of the PR interval may result from a conduction delay in the atrium, atrioventricular node, and/or His-Purkinje system. Although prolongation of the PR interval usually involves a conduction delay in the atrioventricular node, it is frequently accompanied by a conduction delay in the atrium as well. ${ }^{11,12)}$ The total atrial conduction time was recently found to be a very strong predictor of $\mathrm{AF} .^{13,14)}$ The prolonged PR interval probably also reflects a prolonged atrial conduction time that is likely caused by atrial fibrosis and atrial dilatation, which may be involved in the substrate for $\mathrm{AF}^{4)}$ In addition, a prolonged PR interval results in delayed and ineffective mitral valve closure and diastolic mitral regurgitation, ${ }^{15)}$ especially when the PR interval exceeds $230 \mathrm{~ms},{ }^{16}$ ) which promotes increased LA pressure. Increased LA pressure has been shown to be associated with AF. ${ }^{17,18)}$

In patients with a prolonged PR interval, most of the pathogenic factors causing AF remain after ablation. It is therefore reasonable to believe that a prolonged PR interval lowers the success rate of catheter ablation for AF. In this study, we sought to address the outcome of catheter ablation for AF in patients with a prolonged PR interval. To the best of our knowledge, our data show for the first time that a prolonged PR interval increases the incidence of recurrence after catheter ablation for paroxysmal AF in both univariate and multivariate analyses. Several mechanisms may account for the relationship between a prolonged PR interval and recurrence of AF. First, the prolonged PR interval probably reflects underlying atrial disease with atrial fibrosis or atrial dilatation. Atrial fibrosis ${ }^{19-21)}$ and atrial dilatation ${ }^{22-24)}$ are associated with increased recurrence of AF after catheter ablation. Second, a prolonged PR interval results in increased LA pressure, delayed and ineffective mitral valve closure, and diastolic mitral regurgitation. ${ }^{15)}$ Fur- 
thermore, in patients with a very long PR interval, a short coupling between ventricular and atrial activation can also occur, which may cause atrial contraction against closed AV valves. These factors contribute to increased LA pressure. Geske, et al reported that increased LA pressure was associated with increased LA size. ${ }^{25)}$ The LA size is a well-known predictor of recurrence after catheter ablation for $\mathrm{AF}^{22-24)}$ In addition, mechanoelectric feedback ${ }^{26)}$ and proliferation of arrhythmogenic fibroblasts ${ }^{27,28)}$ secondary to increased LA pressure may also play a role in promoting recurrence of AF. Third, a prolonged PR interval is associated with endothelial dysfunction. Recent studies showed that PR interval prolongation, even in the conventionally normal range, is independently associated with endothelial dysfunction. ${ }^{29,30)}$ Endothelial dysfunction is an important predictor for recurrence of AF after catheter ablation. $^{22)}$

In the present study, patients in the PPR group had a larger LA size than did patients in the NPR group. As mentioned above, LA size is a well-known predictor of recurrence of AF after catheter ablation. ${ }^{22-24)}$ However, after adjusting for the LA diameter, a prolonged PR interval was identified as an independent predictor of recurrence of AF. This may be explained by a recent study ${ }^{19)}$ showing that whereas patients with a severely enlarged LA may be accurately identified as being at high risk for $\mathrm{AF}$ recurrence, patients with mild to moderate LA enlargement show varying responses to catheter ablation. Another likely explanation for this is that when LA dilation inclines to asymmetry, the LA volume may be superior to the LA diameter in terms of predicting recurrence of $\mathrm{AF}^{31,32)}$

As mentioned above, the patients with the prolonged PR interval probably suffered from atrial disease with atrial fibrosis or atrial dilatation, which may be involved in the substrate for AF. Therefore, it seemed to be reasonable that the paroxysmal AF patients with the prolonged PR interval have higher recurrence of AF after single circumferential PV ablation. For these patients, circumferential PV ablation alone may be insufficient. However, whether additional substrate ablation strategies such as left atrial linear lesions or ablation of complex fractionated atrial electrograms are required for these patients to achieve a higher success rate was not determined in this study, and this needs further studies.

Several limitations of this study should be noted. First, because the PR interval was measured from the surface electrocardiogram as opposed to an intracardiac recording, the relative contributions of delays at different conduction sites could not be assessed. Second, because atrial fibrosis was not assessed using delayed enhancement magnetic resonance imaging $^{20)}$ or 2-dimensional echocardiography derived integrated backscatter ${ }^{19)}$ for all patients, whether the patients with a prolonged PR interval suffered from atrial fibrosis was not determined in this study. Third, the autonomic nervous system, which may be closely related to PR interval and cannot be tested in this study, should warrant further investigation. Fourth, $\mathrm{PV}$ reconnection is a known cause of AF recurrence. ${ }^{33,34)}$ The rate of PV isolation did not differ between the PPR and NPR groups, but this study did not determine whether a prolonged PR interval was associated with an increased likelihood of PV reconnection because it is not clear how many patients with $\mathrm{AF}$ recurrence had PV reconnections in two groups. Finally, the diagnosis of recurrence was based on symptoms, ECG, and Holter-ECG findings. The rate of recurrence of AF might have been underestimated because some patients were asymptomatic.

In conclusion, this study is the first to report that a prolonged PR interval is an independent predictor of recurrence of $\mathrm{AF}$ in patients who have undergone catheter ablation.

\section{REFERENCES}

1. Wilke T, Groth A, Mueller S, et al. Incidence and prevalence of atrial fibrillation: an analysis based on 8.3 million patients. Europace 2013 ; 15 : 486-93.

2. Chiang CE, Naditch-Brûlé L, Murin J, et al. Distribution and risk profile of 3 paroxysmal, persistent, and permanent atrial fibrillation in routine clinical practice: insight from the real-life global survey evaluating patients with atrial fibrillation international registry. Circ Arrhythm Electrophysiol 2012; 5: 632-9.

3. Piccini JP, Hammill BG, Sinner MF, et al. Incidence and prevalence of atrial fibrillation and associated mortality among Medicare beneficiaries, 1993-2007. Circ Cardiovasc Qual Outcomes 2012; 5: 85-93.

4. Nielsen JC, Thomsen PE, Højberg S, et al. Atrial fibrillation in patients with sick sinus syndrome: the association with PQ-interval and percentage of ventricular pacing. Europace 2012; 14: 682-9.

5. Cheng S, Keyes MJ, Larson MG, et al. Long-term outcomes in individuals with prolonged PR interval or first-degree atrioventricular block. JAMA 2009; 301: 2571-7.

6. Tang RB, Dong JZ, Liu XP, et al. Obstructive sleep apnoea risk profile and the risk of recurrence of atrial fibrillation after catheter ablation. Europace 2009; 11: 100-5.

7. Tang RB, Liu DL, Dong JZ, et al. High-normal thyroid function and risk of recurrence of atrial fibrillation after catheter ablation. Circ J 2010; 74: 1316-21.

8. Liu X, Dong J, Mavrakis HE, et al. Achievement of pulmonary vein isolation in patients undergoing circumferential pulmonary vein ablation: A randomized comparison between two different isolation approaches. J Cardiovasc Electrophysiol 2006; 17: 126370.

9. Tang RB, Dong JZ, Liu XP, et al. Metabolic syndrome and risk of recurrence of atrial fibrillation after catheter ablation. Circ J 2009; 73: 438-43.

10. Schnabel RB, Sullivan LM, Levy D, et al. Development of a risk score for atrial fibrillation (Framingham Heart Study): a community-based cohort study. Lancet 2009; 373: 739-45.

11. Schwartzman D. Atrioventricular block and atrioventricular dissociation. In: Zipes D, Jalife J, eds. Cardiac Electrophysiology: From Cell to Bedside. 4th ed. Philadelphia, PA: Saunders, 2004: 485-9.

12. Daubert JC, Pavin D, Jauvert G, Mabo P. Intra- and interatrial conduction delay: implications for cardiac pacing. Pacing Clin Electrophysiol 2004; 27: 507-25. (Review)

13. Bertini M, Borleffs CJ, Delgado V, et al. Prediction of atrial fibrillation in patients with an implantable cardioverter-defibrillator and heart failure. Eur J Heart Fail 2010; 12: 1101-10.

14. De Vos CB, Weijs B, Crijns HJ, et al. Atrial tissue Doppler imaging for prediction of new-onset atrial fibrillation. Heart 2009; 95 : $835-40$.

15. Schnittger I, Appleton CP, Hatle LK, Popp RL. Diastolic mitral and tricuspid regurgitation by Doppler echocardiography in patients with atrioventricular block: new insight into the mechanism of atrioventricular valve closure. J Am Coll Cardiol 1988; 11: 838.

16. Ishikawa T, Kimura K, Miyazaki N, et al. Diastolic mitral regurgitation in patients with first-degree atrioventricular block. Pacing Clin Electrophysiol 1992; 15: 1927-31.

17. Lin WS, Prakash VS, Tai CT, et al. Pulmonary vein morphology in patients with paroxysmal atrial fibrillation initiated by ectopic beats originating from the pulmonary veins: implications for catheter ablation. Circulation 2000; 101: 1274-81. 
18. Qi J, Xiao J, Zhang Y, et al. Effects of potassium channel blockers on changes in refractoriness of atrial cardiomyocytes induced by stretch. Exp Biol Med 2009; 234: 779-84.

19. den Uijl DW, Delgado V, Bertini M, et al. Impact of left atrial fibrosis and left atrial size on the outcome of catheter ablation for atrial fibrillation. Heart 2011; 97: 1847-51.

20. Oakes RS, Badger TJ, Kholmovski EG, et al. Detection and quantification of left atrial structural remodeling with delayed-enhancement magnetic resonance imaging in patients with atrial fibrillation. Circulation 2009; 119: 1758-67.

21. Akoum N, Daccarett M, McGann C, et al. Atrial fibrosis helps select the appropriate patient and strategy in catheter ablation of atrial fibrillation: a DE-MRI guided approach. J Cardiovasc Electrophysiol 2011; 22: 16-22.

22. Shin SY, Na JO, Lim HE, et al. Improved endothelial function in patients with atrial fibrillation through maintenance of sinus rhythm by successful catheter ablation. J Cardiovasc Electrophysiol 2011; 22: 376-82.

23. Miyazaki S, Kuwahara T, Kobori A, et al. Preprocedural predictors of atrial fibrillation recurrence following pulmonary vein antrum isolation in patients with paroxysmal atrial fibrillation: longterm follow-up results. J Cardiovasc Electrophysiol 2011; 22: 6215 .

24. Akutsu Y, Kaneko K, Kodama Y, et al. Association between left and right atrial remodeling with atrial fibrillation recurrence after pulmonary vein catheter ablation in patients with paroxysmal atrial fibrillation: a pilot study. Circ Cardiovasc Imaging 2011; 4: 524 31.

25. Geske JB, Sorajja P, Nishimura RA, Ommen SR. The relationship of left atrial volume and left atrial pressure in patients with hypertrophic cardiomyopathy: an echocardiographic and cardiac cathe- terization study. J Am Soc Echocardiogr 2009; 22: 961-6.

26. Yamazaki M, Filgueiras-Rama D, Berenfeld O, Kalifa J. Ectopic and reentrant activation patterns in the posterior left atrium during stretch-related atrial fibrillation. Prog Biophys Mol Biol 2012; 110: 269-77.

27. Rohr S. Arrrhythmogenic implications of fibroblast-myocyte interactions. Circ Arrhythm Electrophysiol 2012; 5: 442-52. (Review)

28. Wilber DJ. Fibroblasts, focal triggers, and persistent atrial fibrillation: is there a connection? Circ Arrhythm Electrophysiol 2012; 5: 249-51.

29. Chan $\mathrm{YH}$, Siu CW, Yiu KH, et al. Abnormal vascular function in PR-interval prolongation. Clin Cardiol 2011; 34: 628-32.

30. Chan YH, Siu CW, Yiu KH, et al. Prolongation of PR interval is associated with endothelial dysfunction and activation of vascular repair in high-risk cardiovascular patients. J Interv Card Electrophysiol 2013; 37: 55-61.

31. Delgado V, Vidal B, Sitges M, et al. Fate of left atrial function as determined by real-time three-dimensional echocardiography study after radiofrequency catheter ablation for the treatment of atrial fibrillation. Am J Cardiol 2008; 101: 1285-90.

32. Tops LF, Bax JJ, Zeppenfeld K, Jongbloed MR, van der Wall EE, Schalij MJ. Effect of radiofrequency catheter ablation for atrial fibrillation on left atrial cavity size. Am J Cardiol 2006; 97: 1220-2.

33. Yamada T, Yoshida N, Murakami Y, et al. Vagal modification can be a valid predictor of late recurrence of paroxysmal atrial fibrillation independent of the pulmonary vein isolation technique. Circ J 2009; 73: 1606-11.

34. Noguchi H, Kumagai K, Yasuda T, Ogawa M, Tojo H, Saku K. Conduction recovery after pulmonary vein isolation for atrial fibrillation. Circ J 2005; 69: 65-8. 